\title{
Manufacture and final tests of the LSST monolithic primary/tertiary mirror
}

\author{
H. M. Martin ${ }^{\mathrm{a}}$, J. R. P. Angel ${ }^{\mathrm{a}}$, G. Z. Angeli ${ }^{\mathrm{b}}$, J. H. Burge ${ }^{\mathrm{c}}$, W. Gressler ${ }^{\mathrm{b}}$, D. W. Kim ${ }^{\mathrm{c}}$, \\ J. S. Kingsley ${ }^{\mathrm{a}}$, K. Law ${ }^{\mathrm{a}}$, M. Liang ${ }^{\mathrm{b}}$, D. Neill ${ }^{\mathrm{b}}$, J. Sebag ${ }^{\mathrm{b}}$, P. A. Strittmatter ${ }^{\mathrm{a}}$, M. T. Tuell ${ }^{\mathrm{a}}$, \\ S. C. West ${ }^{a}$, N. J. Woolf ${ }^{\mathrm{a}}$ and B. Xin ${ }^{\mathrm{b}}$ \\ ${ }^{a}$ Steward Observatory, University of Arizona, Tucson, AZ 85721, USA \\ ${ }^{\mathrm{b}}$ Large Synoptic Survey Telescope, 950 N Cherry Ave, Tucson, AZ 85719, USA \\ ${ }^{\mathrm{c} C o l l e g e}$ of Optical Sciences, University of Arizona, Tucson, AZ 85721, USA
}

\begin{abstract}
The LSST M1/M3 combines an $8.4 \mathrm{~m}$ primary mirror and a $5.1 \mathrm{~m}$ tertiary mirror on one glass substrate. The combined mirror was completed at the Richard F. Caris Mirror Lab at the University of Arizona in October 2014. Interferometric measurements show that both mirrors have surface accuracy better than $20 \mathrm{~nm}$ rms over their clear apertures, in nearsimultaneous tests, and that both mirrors meet their stringent structure function specifications. Acceptance tests showed that the radii of curvature, conic constants, and alignment of the 2 optical axes are within the specified tolerances. The mirror figures are obtained by combining the lab measurements with a model of the telescope's active optics system that uses the 156 support actuators to bend the glass substrate. This correction affects both mirror surfaces simultaneously. We showed that both mirrors have excellent figures and meet their specifications with a single bending of the substrate and correction forces that are well within the allowed magnitude. The interferometers do not resolve some small surface features with high slope errors. We used a new instrument based on deflectometry to measure many of these features with sub-millimeter spatial resolution, and nanometer accuracy for small features, over $12.5 \mathrm{~cm}$ apertures. Mirror Lab and LSST staff created synthetic models of both mirrors by combining the interferometric maps and the small highresolution maps, and used these to show the impact of the small features on images is acceptably small.
\end{abstract}

Keywords: LSST, telescopes, optical fabrication, optical testing, aspheres, active optics

\section{INTRODUCTION}

The LSST has a unique 3-mirror design with the $8.4 \mathrm{~m}$ annular primary (M1) and the $5.1 \mathrm{~m}$ tertiary mirror (M3) on the same glass substrate. ${ }^{[1]}$ The combined mirror, shown in Figure 1, is a borosilicate honeycomb mirror that was cast and polished at the Richard F. Caris Mirror Lab. ${ }^{[2]}$ The combined mirror design eliminates the need for active control of several alignment degrees of freedom in the telescope, and increases the stiffness of the annular primary mirror.

The design also adds requirements to the manufacturing process. Co-alignment of the two mirrors on the substrate is critical. Use of active optics to control the shapes of the mirrors in the telescope is a more complex issue when two mirrors share the same substrate. Section 2 of this paper explains the use of active optics in the manufacturing process, and the ways we dealt with the additional constraints imposed by two surfaces that bend together. Section 3 describes the methods we used to polish and measure the mirrors. Section 4 presents the results of the final measurements. Section 5 describes surface features that contribute to small-scale wavefront error, and the analysis that showed their impact on the images is acceptable.

\section{ACTIVE OPTICS IN THE MANUFACTURING PROCESS FOR LSST}

\subsection{Low-order shape errors}

For LSST, bending of M1 and M3 are tightly coupled. The optimization of support forces must take into account the effect on both mirrors. Active optics is important to the manufacturing process as well as operation at the telescope. Even in the controlled lab environment, the substrate changes its shape by hundreds of nanometers due to force changes of several newtons and temperature gradients of tenths of a kelvin across the mirror. We can remove most of the effects by measuring the forces and temperature distribution, and compensating for them based on a finite element model. The

Advances in Optical and Mechanical Technologies for Telescopes and Instrumentation II, edited by Ramón Navarro, James H. Burge, Proc. of SPIE Vol. 9912, 99120X

(C) 2016 SPIE · CCC code: 0277-786X/16/\$18 · doi: 10.1117/12.2234501 
compensation reduces the effects of forces and temperature gradients, but it is not perfect and we sometimes see significant variations in the mirrors' shapes. The variations are almost entirely low-order aberrations that can be eliminated with active optics.
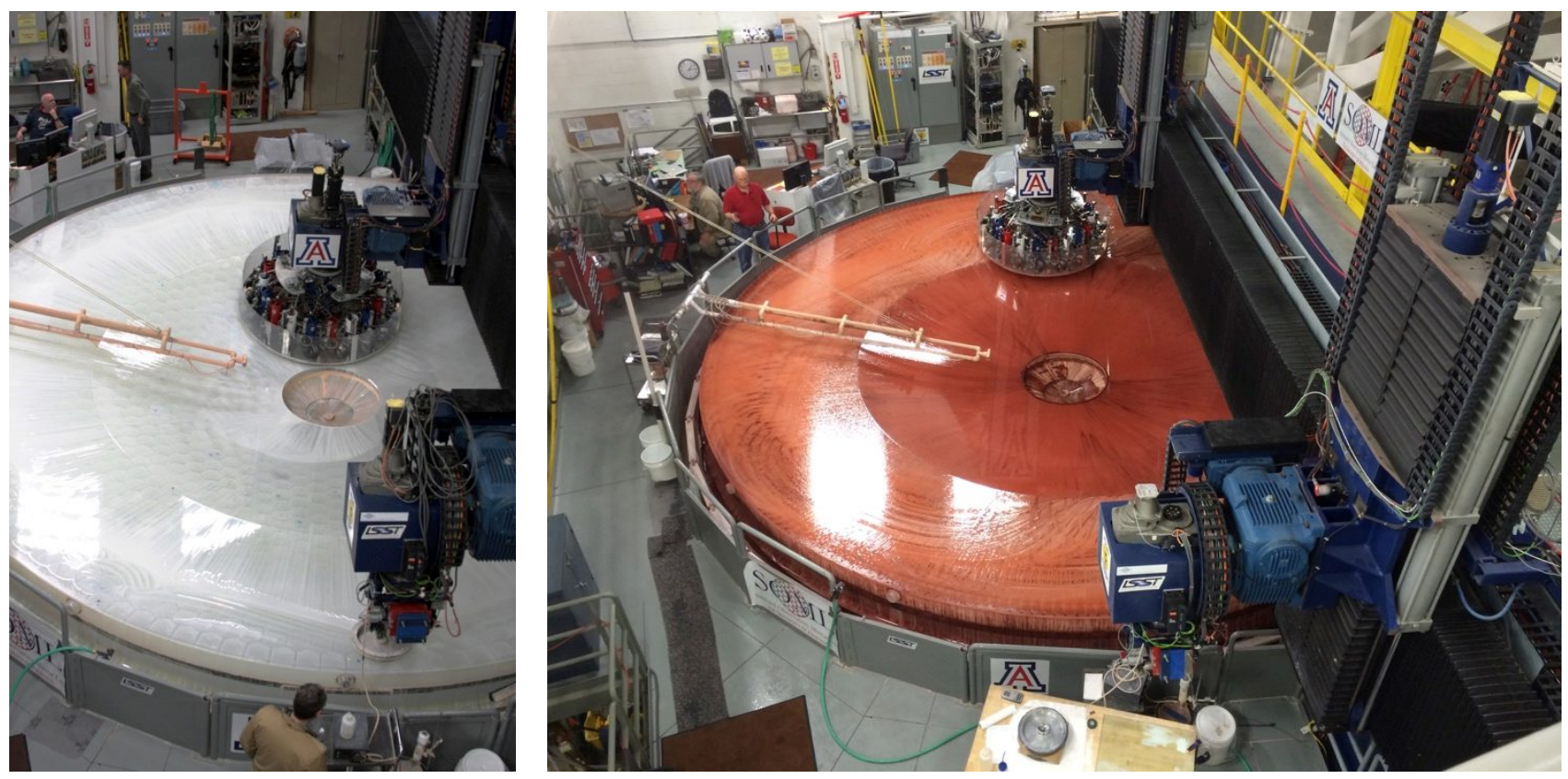

Figure 1. Two views of the combined LSST M1 and M3 being polished. Left: $1.2 \mathrm{~m}$ stressed lap on M3 and $25 \mathrm{~cm}$ orbital lap on M1, with zirconium oxide polishing compound. Right: stressed lap polishing M1 with rouge (iron oxide).

\subsection{Simulating the active-optics correction}

For optical testing in the lab, the mirror is on passive hydraulic supports with the same locations and the same nominal forces as the telescope's active support system at zenith pointing. We cannot apply controlled bending with the passive supports, so we simulate the active correction that will be made at the telescope. We have previously demonstrated good agreement between the simulation and physical bending of the mirror for the MMT, Magellan and LBT primary mirrors. ${ }^{[3]-[7]}$ The figure specification for the mirrors applies to the residual error after this simulated correction. In order to limit the range of force used to correct for manufacturing errors, the magnitude of forces in the simulated correction is limited to $20 \mathrm{~N}$ rms over the 156 actuators.

The simulated active correction uses the 156 actuator influence functions computed from a finite element model. An influence function is defined as the change in the optical surface measurements of both mirrors, due to a unit change in force at an actuator. (The other actuators' forces also change in order to balance the net force and moments.) The model gives the vector displacement of each point on the surface of both mirrors. The quantity we measure optically is the displacement normal to each surface, so we project the vector displacements onto the normal for each surface. This causes the influence functions to be discontinuous at the M1-M3 boundary. The optical measurements are insensitive to piston and tilt of each surface, so we ignore those components of the surface normal displacements. This adds to the discontinuities. Figure 2 shows influence functions for three actuators.

The active-optics correction is a best fit of the influence functions to the measured surface errors on both mirrors. The coefficients of the influence functions give the actuator forces, and the residual error after the fit is the resulting error in the optical surfaces. We limit the magnitude of correction forces, and make the correction more stable, by using a limited number of substrate bending modes as opposed to letting all 156 actuator forces vary freely. Each bending mode is a combination of the 156 influence functions defined so the set of bending modes is orthogonal and ordered from most flexible (maximum ratio of rms surface normal displacement to rms force) to stiffest. For the final figure analysis we used 22 bending modes. Figure 3 shows three bending modes of the combined M1-M3. 

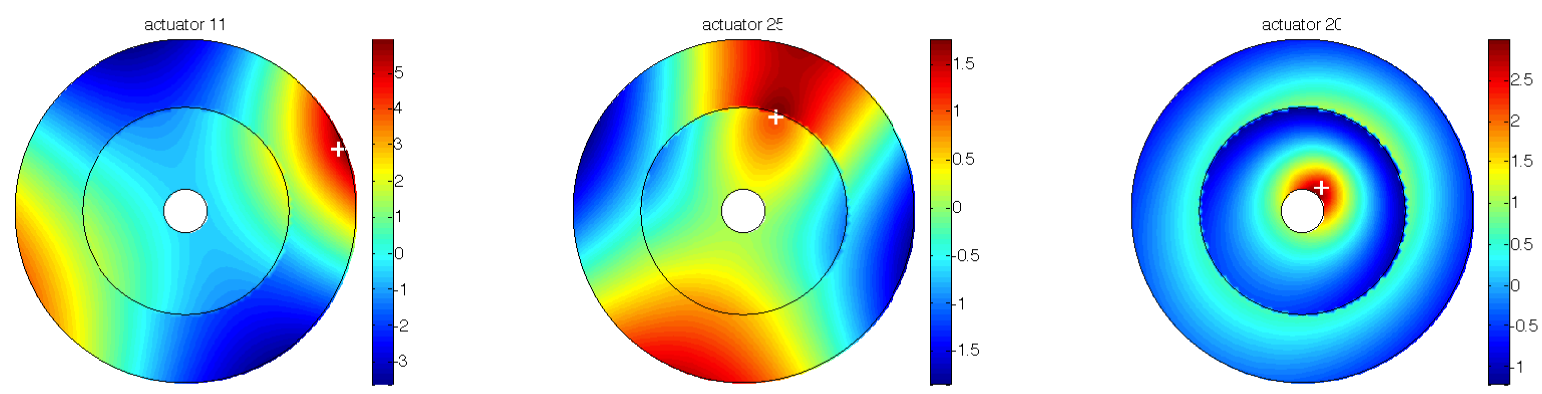

Figure 2. Computed influence functions for the combined M1-M3, for 3 of the 156 actuator. Black circles mark the boundaries of the two mirrors. The color bars give the surface normal displacement in $\mathrm{nm}$ for a $1 \mathrm{~N}$ force at the indicated actuator. The influence functions are broad rather than localized because the forces that balance net force and moment are distributed over all actuators.
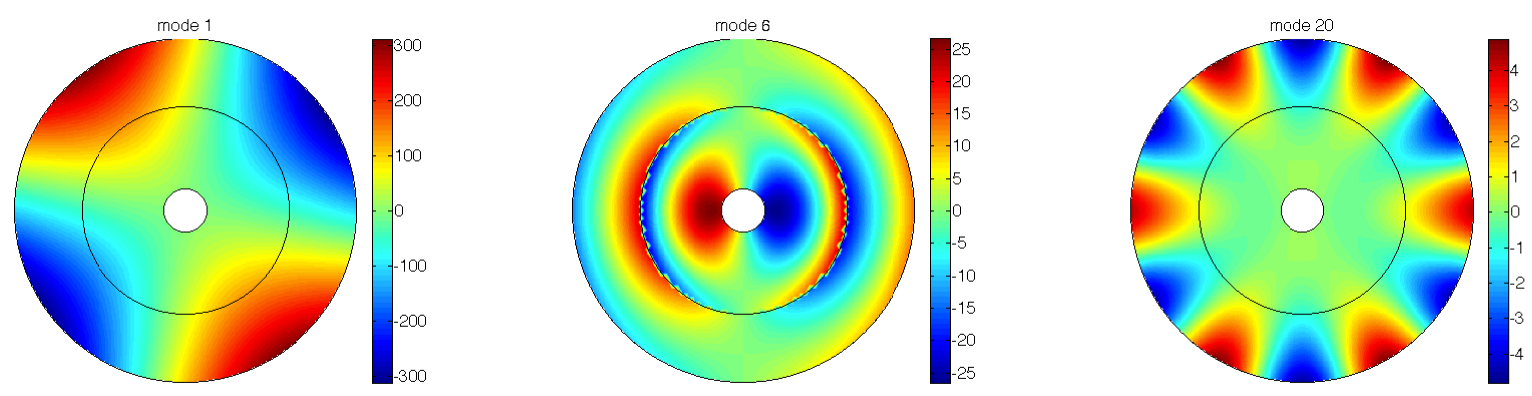

Figure 3. Three bending modes computed for the combined M1-M3. The non-axisymmetric modes come in orthogonal pairs. The color bars give the surface normal displacement in $\mathrm{nm}$ for a force pattern with $1 \mathrm{~N}$ rms variation over the 156 actuators. These three modes span almost two orders of magnitude in stiffness.

The simulation starts with measured wavefronts for M1 and M3, with the substrate assumed to be in the same mechanical and thermal state for both measurements. The optimization of support forces takes account of the effects on both mirrors. In the end, we had to show that both mirrors meet their figure specifications with a single set of correction forces. The requirements for both mirrors are given in terms of structure functions, which specify the surface accuracy as a function of spatial scale.

The fit of bending modes allows any relative weighting of the M1 and M3 surfaces. Regardless of the weighting used, the result is a set of forces that brings both M1 and M3 into compliance with their figure specifications. We chose to tilt the weighting entirely to M1, minimizing the surface error on M1 and simply keeping track of the resulting change in the M3 surface. We did this because M1 is more flexible (defined as the ratio of rms displacement to rms force) than M3, so the bending is more effective on M1. We finished M1 before M3, which effectively froze the bending at the amount that eliminated low-order shape errors in M1. With the bending forces fixed, we polished out the resulting error on M3.

\section{METHODS OF POLISHING AND TESTING}

\subsection{Polishing}

Polishing of both mirrors covered the period April 2012 - October 2014. M1 was finished first, in April 2014, and M3 was finished in October 2014. Acceptance tests were conducted from October 2014 to February 2015.

We polished the optical surface with a combination of techniques. Three types of tools were used:

1. The $1.2 \mathrm{~m}$ stressed lap ${ }^{[8]}$, a stiff tool that bends actively to follow the varying curvature across the surface. It was used either with bare pitch or with synthetic polishing pads. Later figuring with the stressed lap used a pitch diameter of only $80 \mathrm{~cm}$. 
2. Rigid-conformal (RC) laps ${ }^{[9],[10]}$ containing a layer of non-Newtonian fluid between a stiff metal disk and (on the polishing side) a thin rubber diaphragm covered with synthetic polishing pads. We used RC laps with diameters of $35 \mathrm{~cm}, 25 \mathrm{~cm}$ and $12 \mathrm{~cm}$. All were used with an orbital polisher.

3. Small pitch laps covered with synthetic polishing pads, also used with the orbital polisher. We used pitch laps with diameters of $10 \mathrm{~cm}, 7 \mathrm{~cm}$ and $5 \mathrm{~cm}$.

We used different polishing modes for the stressed lap and the smaller passive laps. The stressed lap travels over the full mirror surface many times in a polishing run, with dwell and lap rotation varying to remove selectively based on the current figure error. We sometimes varied the polishing pressure (lap force) and pressure gradients (moments applied to the lap) in proportion to surface error. The stressed lap is most valuable for large-scale figuring and smoothing on small scales. The orbital laps are used in a traditional computer-controlled polishing mode with dwell varying over a large dynamic range to give selective removal. This method is very deterministic and effective at controlling mid-scale structure. ${ }^{[10]}$

For M1, most of the final figuring was done using the $80 \mathrm{~cm}$ stressed lap with pitch, and the $25 \mathrm{~cm}$ RC lap. For M3, when the mirror was nearly finished we noticed that a layer of nylon in the stressed lap was failing at multiple bond joints. (The nylon takes up the sag difference between the flat plate and the curved mirror. It was thicker for M3 with $R=$ $8.3 \mathrm{~m}$, and therefore under greater stress due to plate bending, than for any mirror we had previously polished.) We did not use the stressed lap after that time. The final figuring was done with the $25 \mathrm{~cm}$ and $12 \mathrm{~cm} \mathrm{RC}$ laps and the $10 \mathrm{~cm}$ and $5 \mathrm{~cm}$ pitch laps, all with polishing pads. Losing the stressed lap near the end of processing M3 had an effect on the final surface quality, discussed in Section 5. The stressed lap was the most efficient tool for smoothing out small-scale features. With the more flexible RC laps and the small pitch laps, we were able to improve the mid-scale figure but did not achieve as much removal or smoothing as we would have with the stressed lap.

\subsection{Testing}

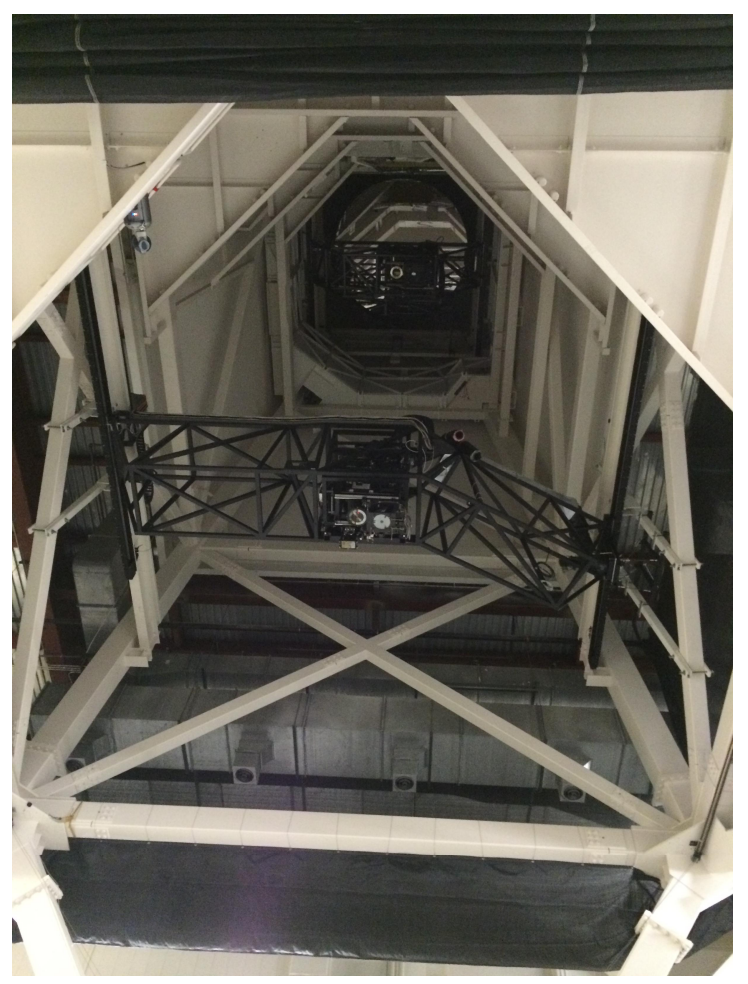

Figure 4. View of LSST test optics looking up into test tower from edge of mirror. Lower (closer) bridge contains M3 null test and upper bridge contains M1 test.
Ref. [11] describes the measurements in detail. We used an interferometer and null corrector for center-of-curvature tests of each mirror. The test systems were at different stations because of the different radii of curvature, $R=19.8 \mathrm{~m}$ for M1 and $8.3 \mathrm{~m}$ for M3. The M3 test system was between M1 and the M1 test system, so its bridge caused a partial obscuration of M1 if the mirrors were measured at the same time. The M3 bridge was deployable so it could be removed to allow an unobscured test of M1. Figure 4 shows the two test systems in position for measurements in the test tower.

We used both simultaneous and sequential tests to show that M1 and M3 meet their figure specifications with the glass substrate in the same state, i. e. after a single active-optics correction for the mirror substrate, taking account of shape changes for both mirrors. Before simulating the active-optics correction, we corrected the measured shape of each mirror for support force errors and temperature gradients at the time of each measurement, as described in Section 2.1.

We used a nearly simultaneous sequential test to demonstrate that both mirrors fully meet their figure specifications after a single active-optics correction. We measured M1, then inserted the M3 test optics to measure M3, then removed the M3 test optics to measure M1 again (the 1-3-1 test). We averaged the two measurements of M1, combined the result with the single measurement of M3, and used these maps as input to the active-optics correction of the substrate. The three measurements were done in a period of about 90 minutes, so the substrate experienced little change due to varying support forces and temperature gradients. Averaging the initial and 
final measurements of M1 nearly eliminates the linear component of any temporal drifts.

To confirm that the 1-3-1 test accurately represents the shape of both mirrors with the substrate in the same state (same support forces and temperature gradients), we made several simultaneous measurements. The partial obscuration of M1 in the simultaneous test compromised the small-scale accuracy in some parts of the mirror. Changes in the state of the substrate affect the large-scale shape of both mirrors but have essentially no effect on small-scale structure. The simultaneous tests agreed with the 1-3-1 test in the large-scale shape of both mirrors after the active-optics correction, within the tolerance on figure error. They confirmed that both mirrors meet their figure specifications with a single active-optics correction.

The null corrector for M1 is a 2-element Offner null lens. The corrector for M3 is a computer-generated hologram (CGH). We confirmed the accuracy of each null corrector by measuring a validation CGH that mimics a perfect mirror. The validation holograms are designed and manufactured independently of the null correctors; they depend only on the mirror prescriptions. We estimate that the validation holograms are slightly more accurate than the null correctors, so we used them to calibrate the null correctors and applied a small correction to the measurement of each mirror surface.

We used two, largely independent, methods of measuring each mirror's radius of curvature. The primary measurement (the more accurate measurement) used a laser tracker to measure the distance between the mirror and the validation hologram. The secondary measurement used a steel tape to measure the distance. We also had two independent measurements of each mirror's conic constant. Both were based on the measured wavefront error; spherical aberration in the wavefront indicates an error in conic constant. The primary measurement used the measured wavefront after correction based on the validation hologram. The validation hologram is therefore the primary standard for conic constant. The secondary measurement used the measured wavefront determined by the null corrector without the correction. The null corrector is the secondary standard for conic constant.

We measured the positions of the two mirrors' optical axes by rotating the mirror around its mechanical axis defined by the outer diameter. For each mirror, the change in coma between a measurement with the mirror at its standard orientation and a measurement with the mirror rotated $180^{\circ}$ is proportional to the displacement of the optical axis from the mechanical axis.

The two remaining alignment parameters are the axial displacement between the vertices of the two mirrors and the relative tilt of the two surfaces. They were measured by scanning the combined surface with a laser tracker.

\section{RESULTS OF FINAL TESTS}

We made the final interferometric measurements on October 19-22, 2014. Figure 5 shows the surface errors on M1 and M3, before and after the simulated active-optics correction. In order to illustrate the active-optics correction we combine the two data sets (M1 and M3) into a single plot. Three pairs of before and after maps are shown, obtained on different days. The maps before correction show how the figure can change from day to day due to variations in support forces and temperature gradients. But the maps after correction are identical within the tolerance on figure error. In all cases the active-optics correction uses forces are well below the $20 \mathrm{~N}$ rms allowance.

Figure 6 shows the averages of the final maps of M1 and M3 after the active-optics correction. The rms surface errors over the specified apertures are $19 \mathrm{~nm}$ for M1 and $18 \mathrm{~nm}$ for M3. The figure specifications are structure functions, giving the allowed error as a function of spatial scale. We plot the square root of the structure function, defined as the rms wavefront different between pairs of points in the aperture as a function of their separation. Figure 7 shows the structure functions computed from the average maps. In addition to the measured structure function, we show the specification for the mirror figure and a slightly tighter tolerance (not a contractual specification) for the measured figure. The tolerance for measured figure includes a conservative estimate of repeating errors in the test, which would cause us to figure the mirror to an incorrect shape, i. e. the measured figure would be better than the actual figure. The structure function plots show that the surface errors meet the requirements, including the conservative estimate of repeating errors in the test. 

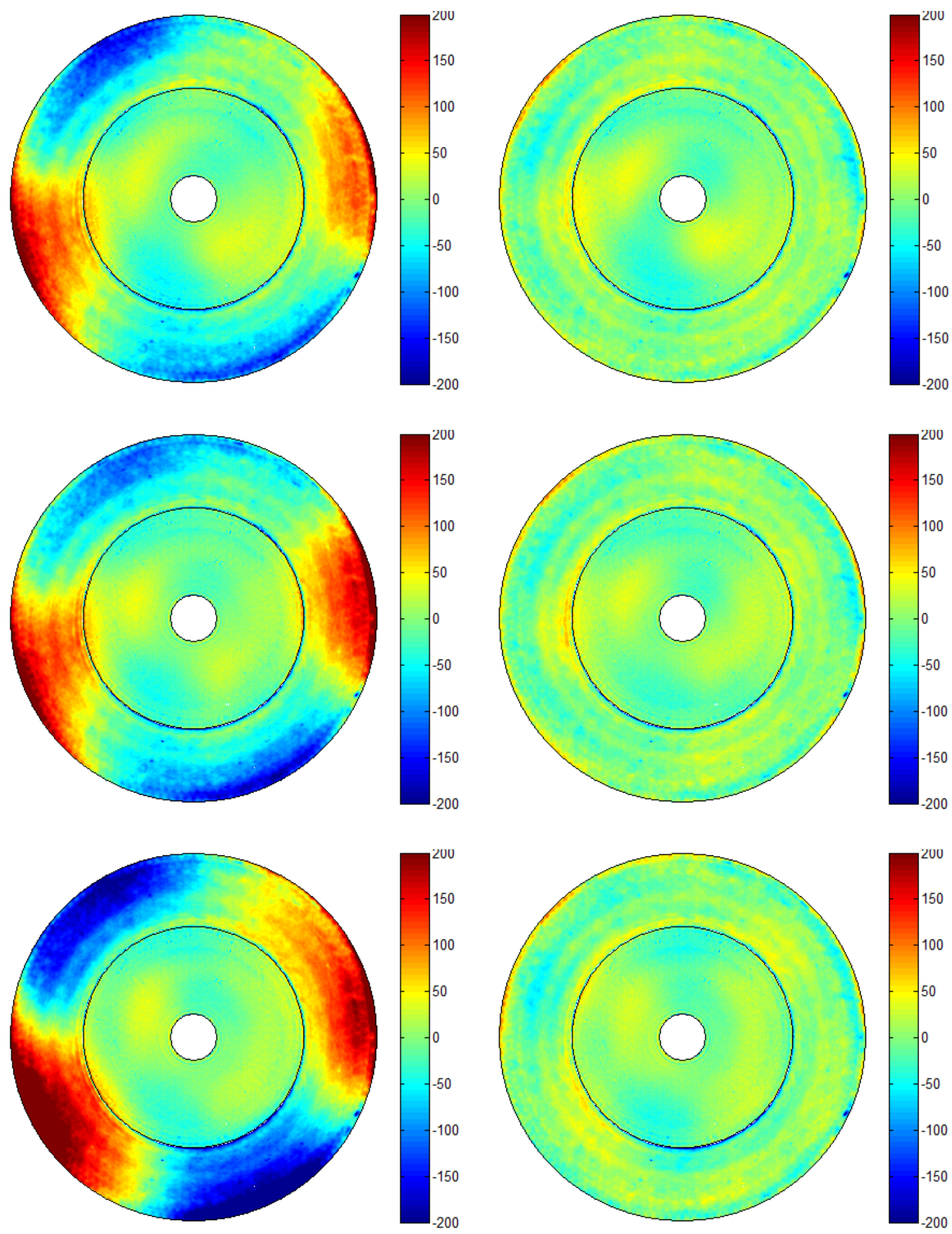

Figure 5. Final set of maps of M1 and M3. Left column is before active-optics correction; right column is after. Each row is measured on a different day. Color bars show surface error in $\mathrm{nm}$. 

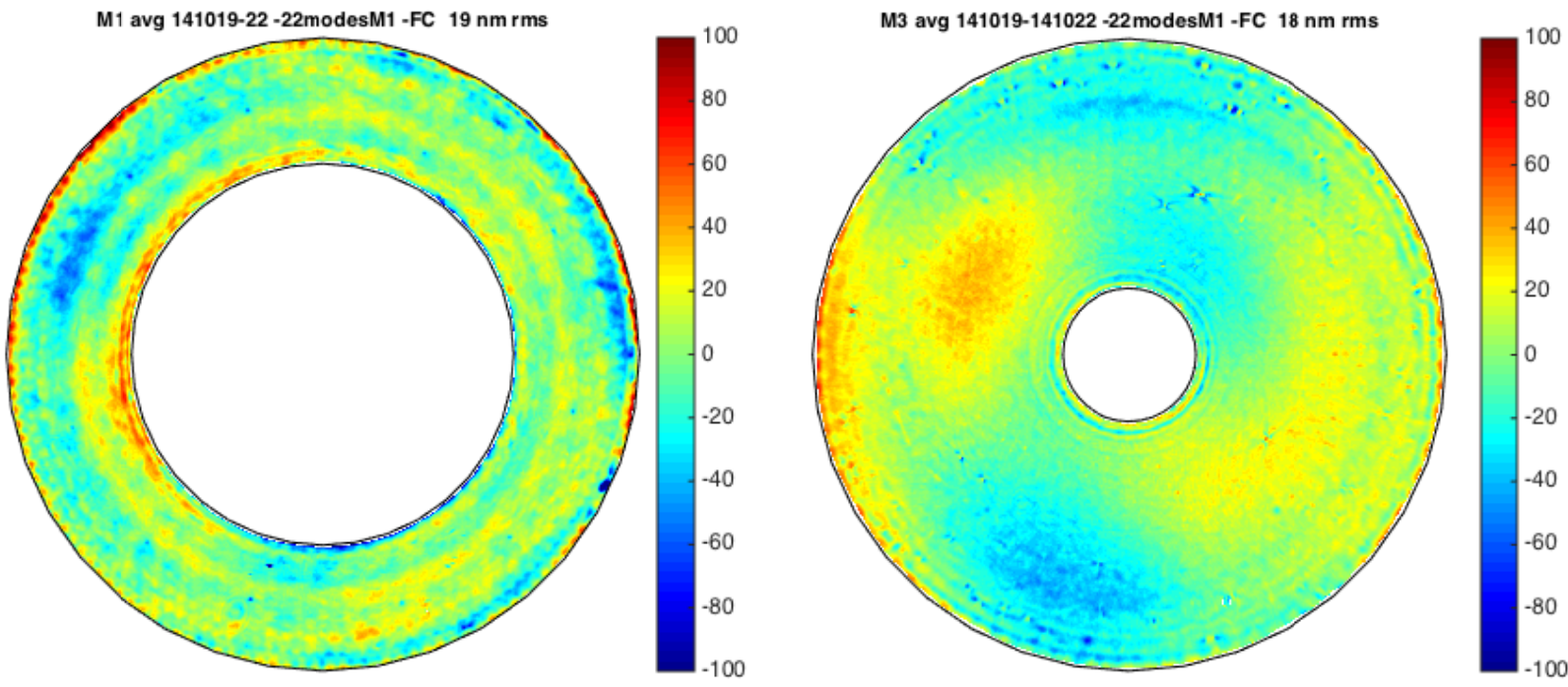

Figure 6. Final average maps of M1 (left) and M3 (right) after active-optics correction. Data are shown only in the clear aperture. Black circles indicate the physical OD and ID. Color bars show surface error in $\mathrm{nm}$.
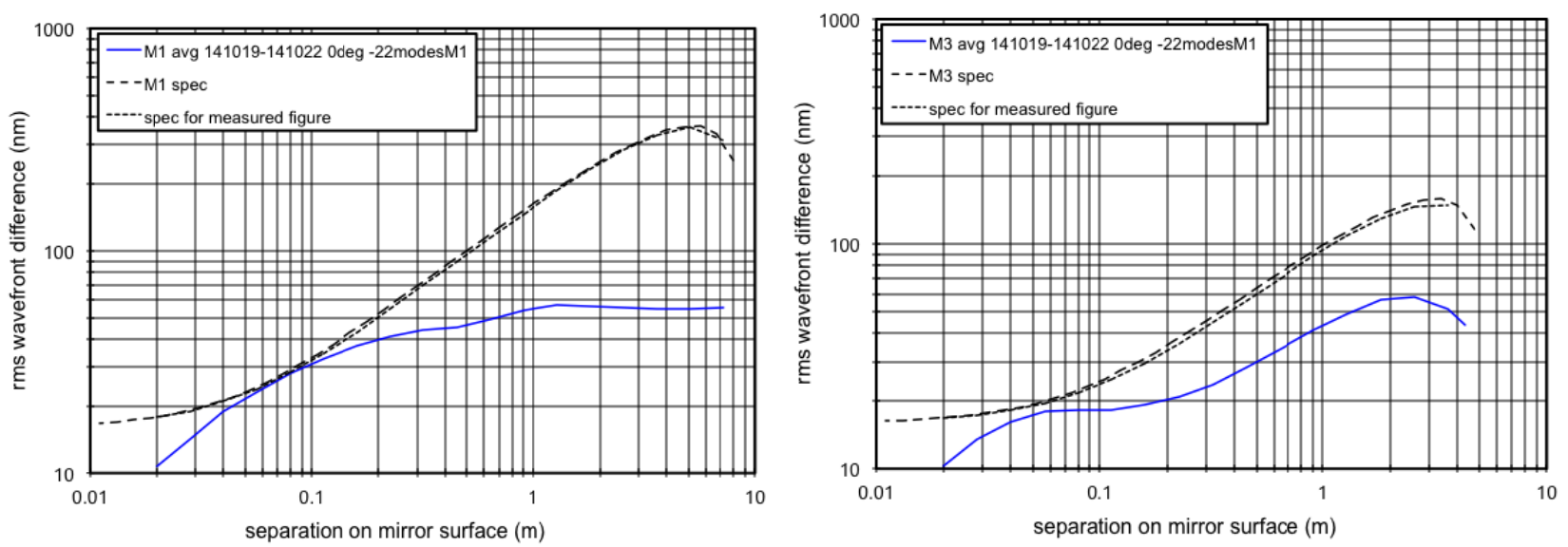

Figure 7. Structure functions for M1 (left) and M3 (right), based on the average maps shown in Figure 6. The upper dashed curves are the mirrors' figure specifications. The lower dashed curves are the tolerances for the measured figure after taking account of possible repeating errors in the test.

We also measured M1 and M3 with the mirror rotated $180^{\circ}$ on October 21-22, 2014. There are systematic changes in the large-scale figure errors when the mirror is at $180^{\circ}$. The mirror support cell is mounted on a different set of points, leading to changes in support forces, and the temperature gradients in the lab are imprinted in the mirror differently. These changes are reduced by the compensation for force and temperature, but the residual effects may amount to tens of nanometers. The overall rms surface errors increase to $24 \mathrm{~nm}$ for M1 and $35 \mathrm{~nm}$ for M3. Large-scale figure errors affect the structure function at large separations, where there is considerable margin between the measured values and the specification. In the $180^{\circ}$ measurements both mirrors meet their figure specifications, including the conservative estimate of repeating errors in the tests.

Table 1 lists the specifications and measured values for the optical prescription and alignment parameters. All parameters meet their specified tolerances. 
Table 1. Optical prescription and alignment parameters. Values for radius of curvature are for the specified temperature of $21^{\circ} \mathrm{C}$. M3 tilt with respect to M1 is defined as the peak-to-valley difference in axial indicator runout on both sides of the M1-M3 boundary. Uncertainties are 2 standard deviations.

\begin{tabular}{|c|c|c|c|c|c|}
\hline parameter & units & nominal value & tolerance & measured value & uncertainty \\
\hline$R$ for M1 & $\mathrm{mm}$ & 19835.5 & 1 & 19835.1 & 0.2 \\
\hline$k$ for M1 & & -1.2150 & 0.0002 & -1.21502 & 0.00011 \\
\hline$R$ for M3 & $\mathrm{mm}$ & 8344.7 & 1 & 8344.1 & 0.1 \\
\hline$k$ for M3 & +0.1550 & 0.0001 & +0.15497 & 0.00005 \\
\hline M1 axis decenter & $\mathrm{mm}$ & 0 & 1 & 0.3 & 0.3 \\
\hline $\begin{array}{c}\text { M3 axis wrt M1 } \\
\text { axis }\end{array}$ & $\mathrm{mm}$ & 0 & 1 & 0.4 & 0.2 \\
\hline M3 tilt wrt M1 & $\mu \mathrm{m}$ & 0 & 100 & 20 & 20 \\
\hline $\begin{array}{c}\text { M1/M3 vertex } \\
\text { spacing }\end{array}$ & $\mathrm{mm}$ & 233.8 & 2 & 234.4 & 0.1 \\
\hline
\end{tabular}

\section{SMALL-SCALE SURFACE FEATURES}

\subsection{Overview of crows' feet}

The LSST mirror surfaces contain small features known as crows' feet that are not resolved in the interferometer data. They are narrow depressions extending from open bubbles in the optical surface. A typical crow's foot has a maximum depth of $1 \mu \mathrm{m}$ and length of a few $\mathrm{cm}$. (This length is the maximum extent over which the feature is detectable in the interferometer map.) About two dozen crows' feet on M3 are larger, with maximum depths of 2-3 $\mu \mathrm{m}$ and lengths of 20$30 \mathrm{~cm}$. Crows' feet result from an interaction between polishing pitch, polishing compound and sharp edges of a bubble, causing high removal as the pitch surface leaves the bubble and comes back onto the optical surface. Many crows' feet are caused by bubbles with diameters $<1 \mathrm{~mm}$, which can be difficult to find until a crow's foot develops. The crow's foot can be seen by viewing a light in reflection off the surface (Figure 8). Once the bubble is found, it can be chamfered and the crow's foot then shrinks with further polishing.

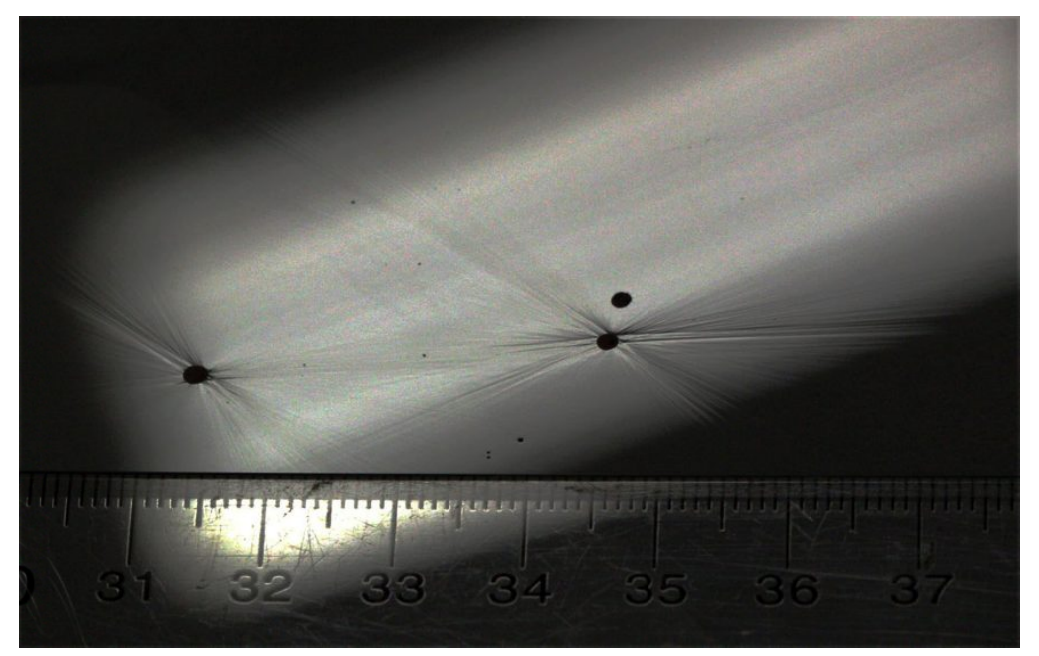

Figure 8. Two of the worst crows' feet on M3, seen as distortion in the reflected image of a fluorescent lamp. What were originally sub- $\mathrm{mm}$ bubbles have been chamfered to diameters of about $1.5 \mathrm{~mm}$. The two bubbles that are the sources of crows' feet are separated by $3 \mathrm{~cm}$. 
To reduce crows' feet to negligible dimensions requires removing several microns of glass along with smoothing action: the surrounding surface must be polished off without significant removal from the low part of the crow's foot. For M1 we achieved enough removal and smoothing to make the crows' feet insignificant, as we have for all previous mirrors, by polishing with the stressed lap. When we experienced a mechanical failure of the stressed lap while polishing M3 (described in Section 3.1), we completed the figuring with smaller tools, both RC laps and pitch laps faced with synthetic polishing pads. None of these tools has as high a removal rate or smoothing rate as the stressed lap. We achieved an excellent figure on large and intermediate scales without removing the crows' feet to the same extent as on M1. M3 therefore has a higher density of crows' feet and they are on average longer and deeper than the crows' feet on M1. Because of the more severe crows' feet on M3, it was necessary to make additional measurements and perform additional analysis to show that the mirrors would meet all performance requirements.

The interferometers for both mirrors have spatial resolution of about $10 \mathrm{~mm}$ at the mirror surface, so they do not resolve the crows' feet well. We implemented a new measuring device, the Slope-measuring Portable Optical Test System $(\mathrm{SPOTS})^{[12]}$, that uses deflectometry to measure the surface with sub-mm resolution over a $12.5 \mathrm{~cm}$ aperture. We measured a representative sample of crows' feet on each mirror with SPOTS and used the data to construct realistic models of both optical surfaces. We then calculated the effect of the crows' feet on the LSST point-spread function. The effect was found to be acceptable. We describe the model and show results of this analysis in the following sections.

\subsection{Measurement of crows' feet}

SPOTS produces a high-resolution map of a small area by measuring slope errors and integrating them. It has a source consisting of a screen that can project patterns onto the surface, and a camera that measures the image of those patterns reflected by the mirror surface. A ray-trace analysis gives the slope errors at the mirror surface. Figure 9 shows the system as a diagram and in use on the LSST mirror. The SPOTS aperture is $12.5 \mathrm{~cm}$ and it samples the mirror surface at $0.18 \mathrm{~mm}$ spacing. Comparison with interferometric tests demonstrates that SPOTS is accurate to about $1 \mathrm{~nm}$ rms for small features. ${ }^{[12]}$

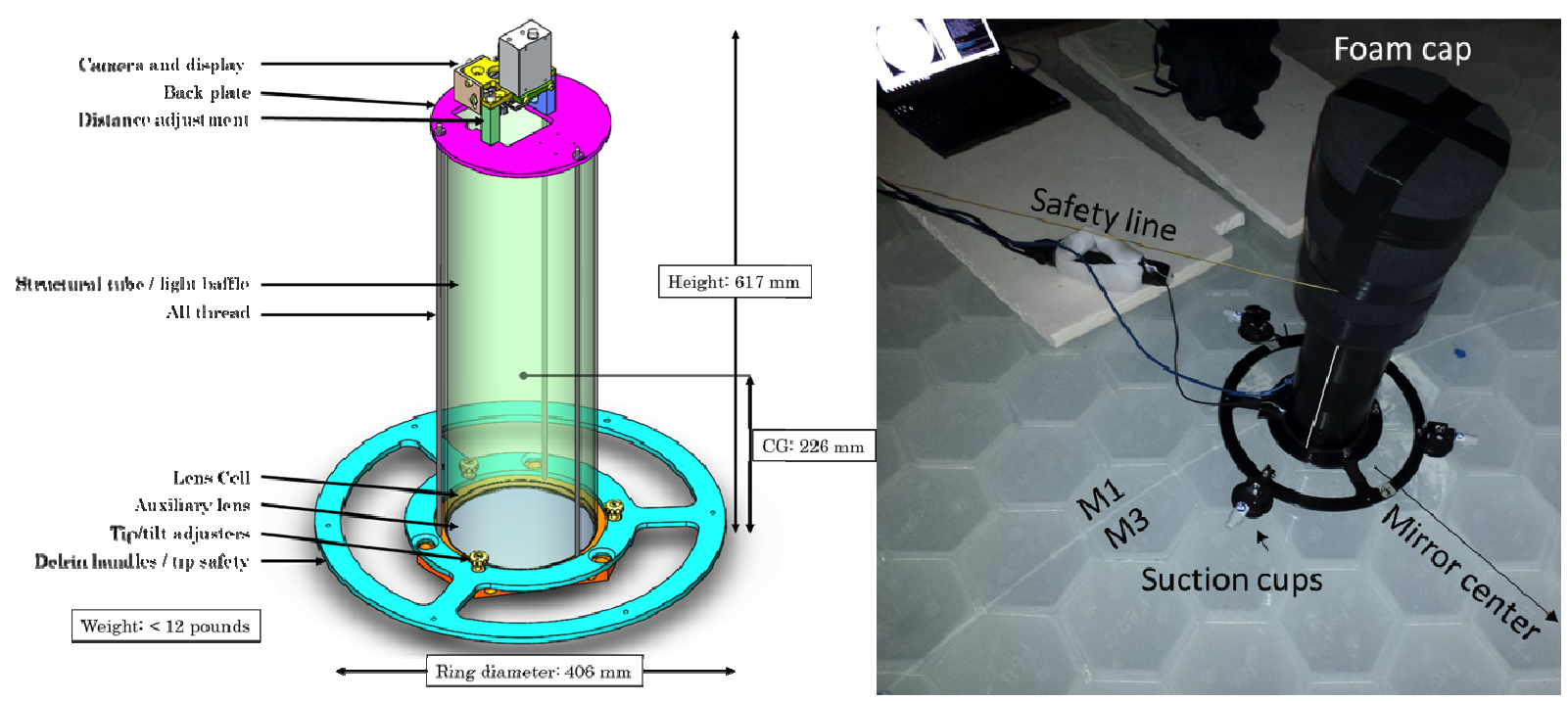

Figure 9. Left: diagram of the SPOTS system. Right: SPOTS set up to measure a feature near the outer edge of M3.

\subsection{Structure of crows' feet}

The structure of a crow's foot is apparent in the interference fringes made with a test plate, Figure 10. The trenches result from repeated motion of the polishing tool across the bubble. Figure 10 also shows the SPOTS map of the same crow's foot. This is a relatively large crow's foot on M3. The visual assessment of its size (region over which one can see a distorted reflection of a background light) is $9 \mathrm{~mm} \times 3 \mathrm{~mm}$. Both the test plate and SPOTS show trenches extending over a larger region. The maximum depth is $1.5 \mu \mathrm{m}$ according to the SPOTS measurement. 

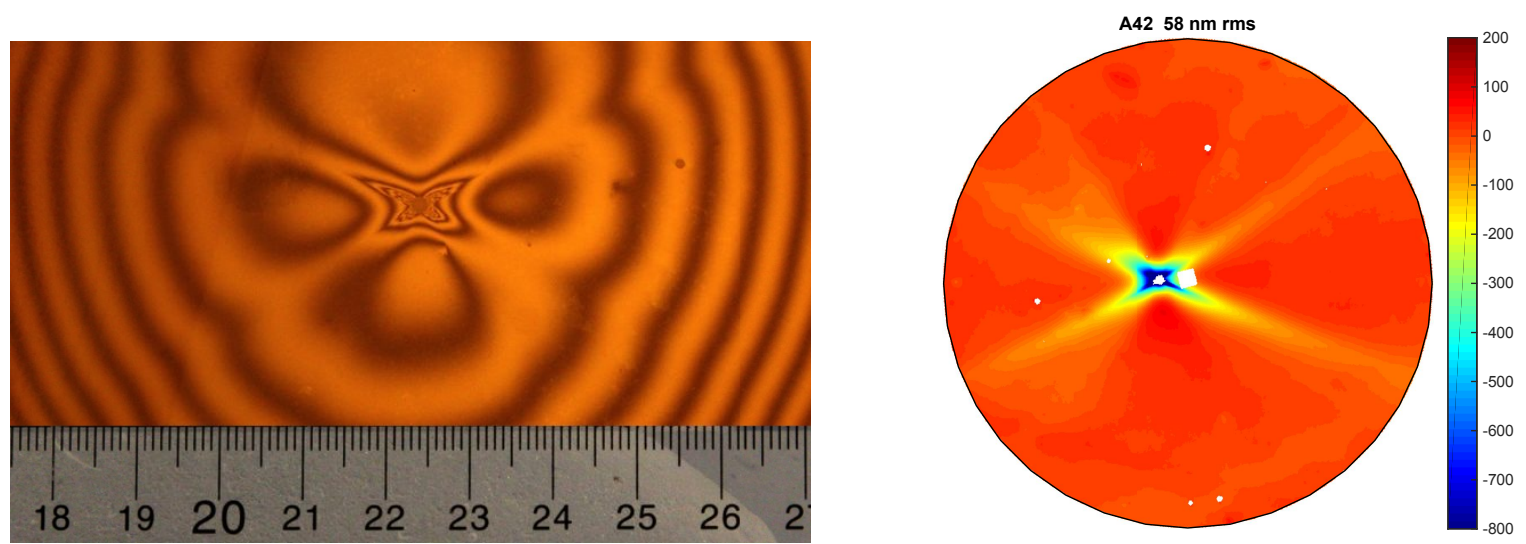

Figure 10. Left: interference fringes from a Fizeau test plate over a large crow's foot on M3. Ruler is labeled in cm. Right: SPOTS map of the same crow's foot. Color bar shows surface error in $\mathrm{nm}$. The SPOTS aperture is $12.5 \mathrm{~cm}$ diameter.

\subsection{Population of crows' feet}

We surveyed the crows' feet on both mirrors visually. The visual assessment is based on the distorted reflection of a fluorescent lamp (Figure 8). The location, length and width of each crow's foot were recorded. The visual length $l$ is defined as the maximum extent over which one can see a distorted image, along any direction across the crow's foot. The survey is nearly complete down to $l=5 \mathrm{~mm}$.

Figure 11 shows the histograms of number vs. visual length, using 1-mm bins for length. The histograms are plotted in a way that makes clear the relative density of crows' feet (number per $\mathrm{m}^{2}$ ) on $\mathrm{M} 1$ and $\mathrm{M} 3$. The vertical axes are plotted so the area in blue on the page is proportional to the density on the mirror surface, taking account of the larger area of M1. The total number of crows' feet with $l \geq 5 \mathrm{~mm}$ is 111 for M1 and 167 for M3. M1 has almost twice the area of M3. While it has $2 / 3$ as many crows' feet as M3, its density of crows' feet is only $37 \%$ that of M3.
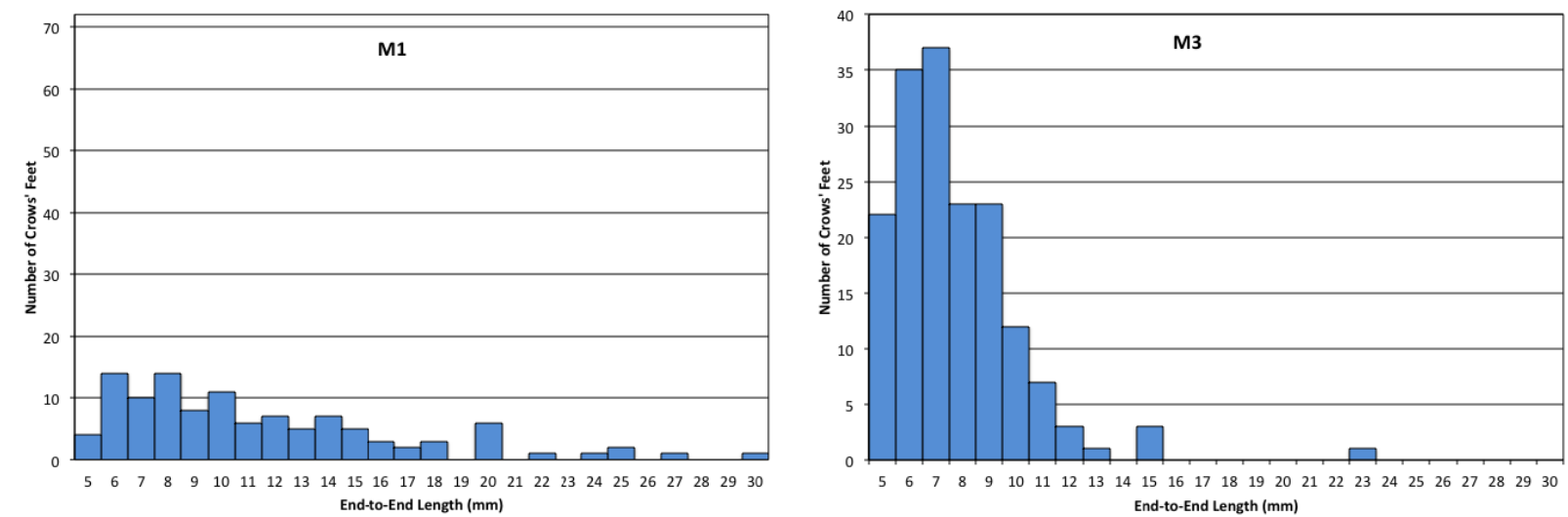

Figure 11. Number of crows' feet vs. length $l$ (based on visual assessment) for M1 (left) and M3 (right).

The distribution of crows' feet on M1 is flatter, with a higher fraction at larger lengths in the visual survey. But the SPOTS measurements show that, for a given $l$, crows' feet on M1 tend to be much smaller than those on M3. Figure 12 illustrates the difference for a typical pair of crows' feet that were assigned the same length in the visual survey. For M3, the extent of significant structure in the SPOTS maps exceeds the visual length by a factor of 10 or more. SPOTS is sensitive to much smaller slope errors than can be seen by eye in the reflected images. For M1, however, the SPOTS maps generally show significant structure extending only 2-3 times the visual length. Most of the M1 crows' feet have negligible slope errors far beyond the region that can be seen by eye. This difference is probably due to the additional stressed-lap polishing of M1. This polishing removed the shallow parts of the crows' feet that extend far from the bubble. 

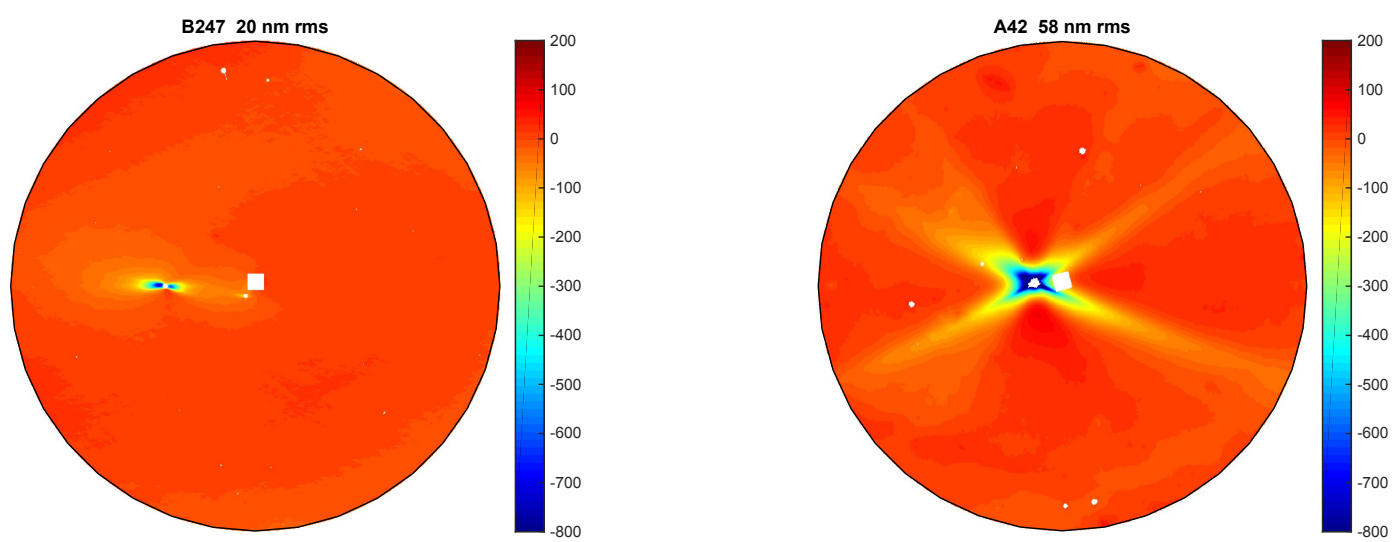

Figure 12. SPOTS maps of a crow's foot in M1 (left) and one in M3 (right), both classified as $9 \mathrm{~mm}$ long in the visual survey. Color bars show surface error in $\mathrm{nm}$. The SPOTS aperture is $12.5 \mathrm{~cm}$ diameter.

\subsection{Synthetic maps including crows' feet}

The structure function specification is based on the wavefront distorted by the atmosphere, and it is appropriate for mirror figure errors whose distribution (area covered vs. magnitude of error) is similar to that of the atmospherically distorted wavefront. A distribution with a small fraction of the area having extreme values of surface error may have a large effect on the structure function even if the effect on the image is small. To assess the impact of crows' feet on telescope performance, we evaluated the point-spread function (PSF) for a model of the mirror surfaces including a highresolution representation of the crows' feet. We used diffraction analysis to compute the PSF and encircled energy for this model.

We used SPOTS to measure a representative sample of 19 crows' feet on M1 and 22 crows' feet on M3. The sample includes crows' feet whose lengths $l$, based on the visual survey, cover the range from $6 \mathrm{~mm}$ to $30 \mathrm{~mm}$. The sample includes all crows' feet with $l \geq 20 \mathrm{~mm}$. For each mirror we made a library of SPOTS maps of crows' feet, covering the full range of lengths.

We created synthetic maps of M1 and M3 by adding SPOTS maps to the interferometer maps. At the location of each crow's foot with $l \geq 5 \mathrm{~mm}$, we added a SPOTS map. If there was a SPOTS map of that particular crow's foot, we used it. If not, we used a SPOTS map from the library for a crow's foot of the same length within $1 \mathrm{~mm}$. Several SPOTS maps contain two crows' feet - these were used only at the actual location of those crows' feet - so the number of SPOTS maps added is less than the number of crows' feet. The synthetic map of M1 has 106 SPOTS maps added to the interferometer map, and the synthetic map of M3 has 163 SPOTS maps added.

We rotated each SPOTS map to the correct orientation before adding it to the interferometer map. To eliminate discontinuities at the edge of the aperture, which would put spurious structure into the PSF, we apodized each SPOTS map by multiplying by $1-[r /(d / 2)]^{2}$, with $d=12.5 \mathrm{~cm}$. This reduced the structure in the crow's foot (slightly, because most crows' feet are near the center of the map), so we rescaled the apodized map to recover the original rms surface error.

Figure 13 shows the M1 interferometer map and the synthetic map of M1. The added SPOTS maps can barely be made out in the synthetic map, and they do not change the rms surface error $(22 \mathrm{~nm})$. Figure 14 shows the M3 interferometer map and the synthetic map of M3. One can easily see the added SPOTS maps, and they change the rms surface error from $19 \mathrm{~nm}$ to $25 \mathrm{~nm}$. 

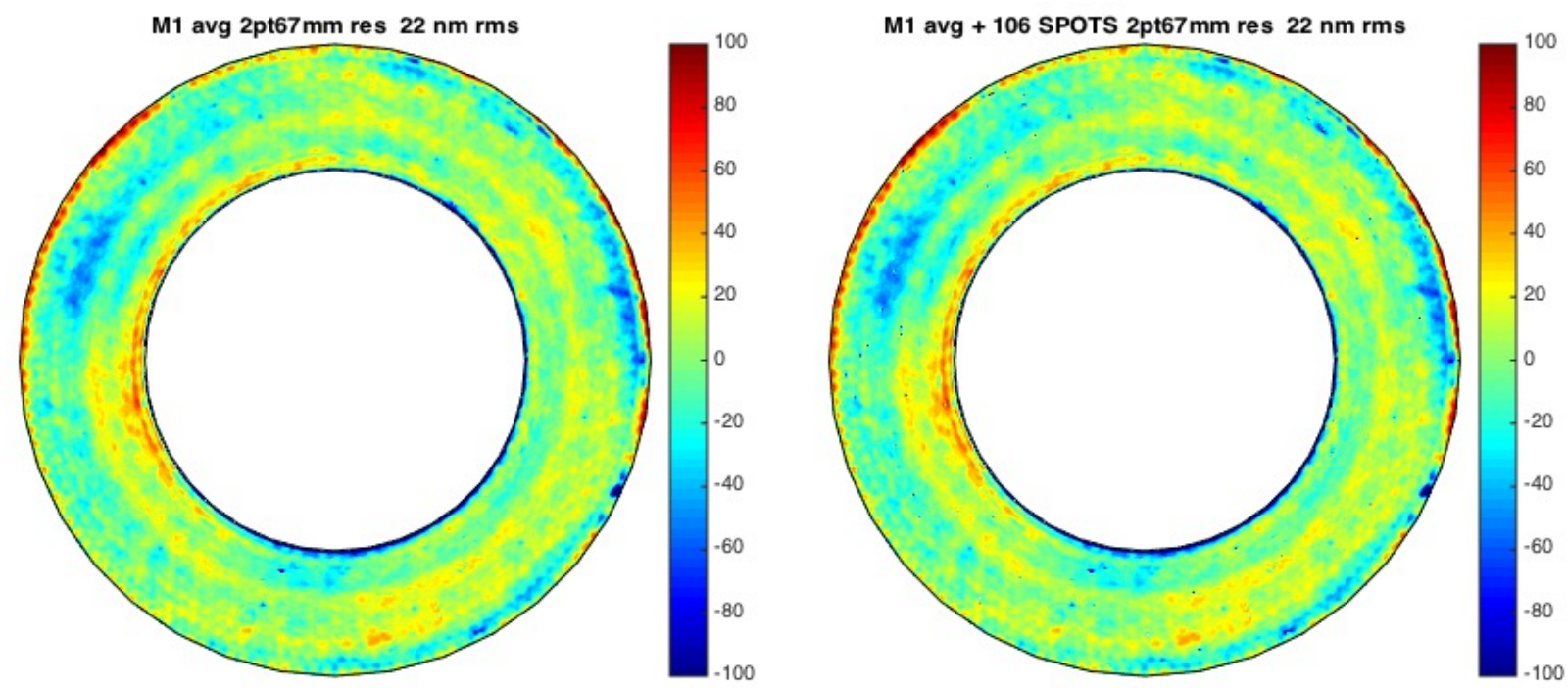

Figure 13. Interferometer map of M1 (left) and synthetic map with 106 SPOTS maps added to the interferometer map (right). Both maps are sampled at $2.67 \mathrm{~mm}$ spacing, but the display resolution is not adequate to show the structure in the SPOTS maps. The maps cover the full aperture, $2.533 \mathrm{~m}<r<4.202 \mathrm{~m}$. The color bars show surface error in $\mathrm{nm}$.
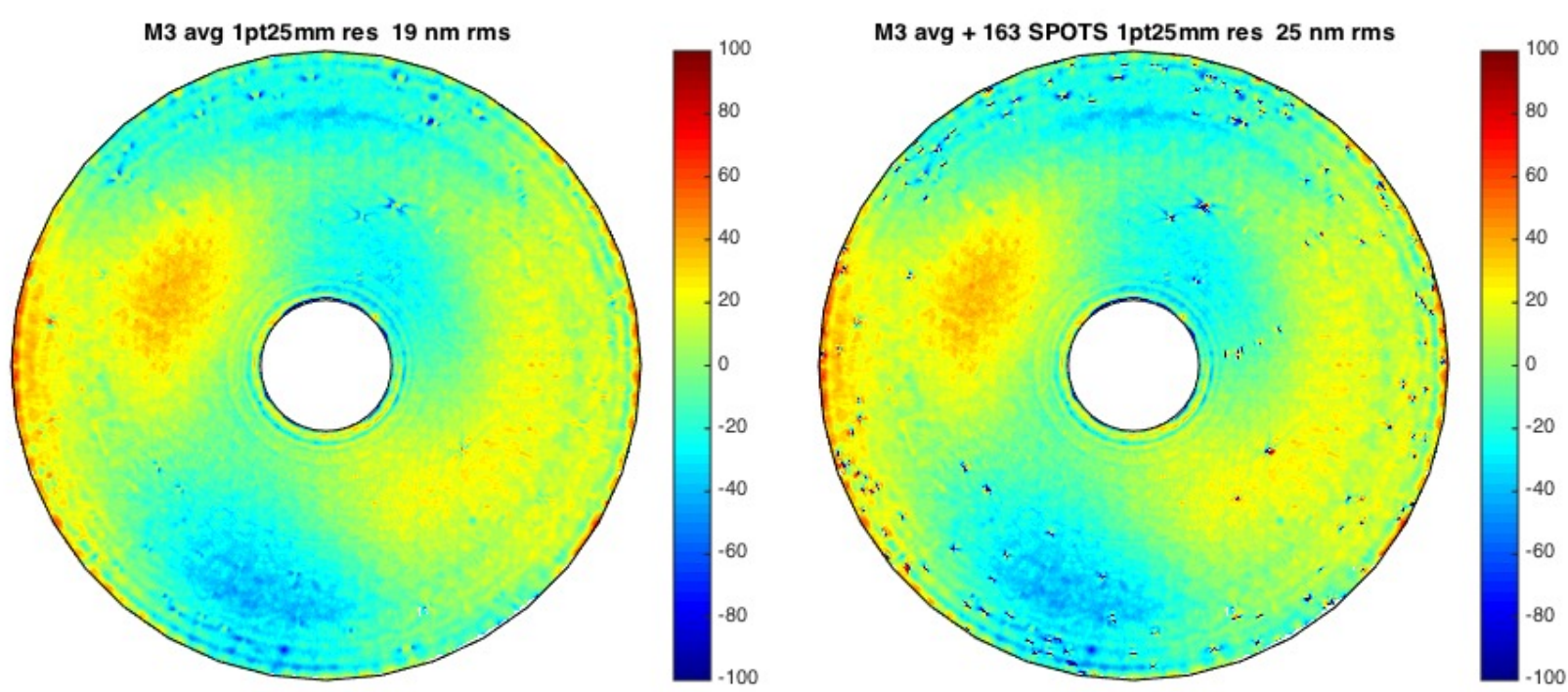

Figure 14. Interferometer map of M3 (left) and synthetic map with 163 SPOTS maps added to the interferometer map (right). Both maps are sampled at $1.25 \mathrm{~mm}$ spacing, equivalent to $2.67 \mathrm{~mm}$ in the pupil, but the display resolution is not adequate to show the structure in the SPOTS maps. The maps cover the full aperture, $0.534 \mathrm{~m}<r<2.533 \mathrm{~m}$.

\subsection{Impact of the crows' feet}

We evaluated the effect of the crows' feet on the image over a 39 arcsecond field, at a wavelength $\lambda=500 \mathrm{~nm}$. We sampled the wavefront error in the pupil at $2.67 \mathrm{~mm}$ spacing, giving a field of $\lambda /(2.67 \mathrm{~mm})=39$ arcsecond. The SPOTS maps have $0.18 \mathrm{~mm}$ sampling, while both interferometers have about $10 \mathrm{~mm}$ sampling on the mirror surface. For M3 this is equivalent to $21 \mathrm{~mm}$ sampling in the pupil. The field of the PSF calculation therefore extends beyond any structure that can come from the interferometer maps, but the SPOTS maps have more than adequate sampling for the PSF calculation.

To compute the PSF at a particular field angle, we added the synthetic maps of M1 and M3, taking account of the mapping of the pupil onto M3, which varies with field angle. We did not include the wavefront error inherent in the 
optical design, which is insignificant in 0.6 arcsecond seeing. We used field angles of $0,1.225^{\circ}$ and $1.75^{\circ}$ based on LSST system requirements. For a field angle of 0 , the pupil covers only a $3.6 \mathrm{~m}$ diameter on M3, missing the outer part where the density of crows' feet is highest. This can be seen in Figure 15 . The $1.75^{\circ}$ field angle includes the most area near the edge of M3, so it is most affected by the crows' feet. All the results we present are for a field angle of $1.75^{\circ}$.
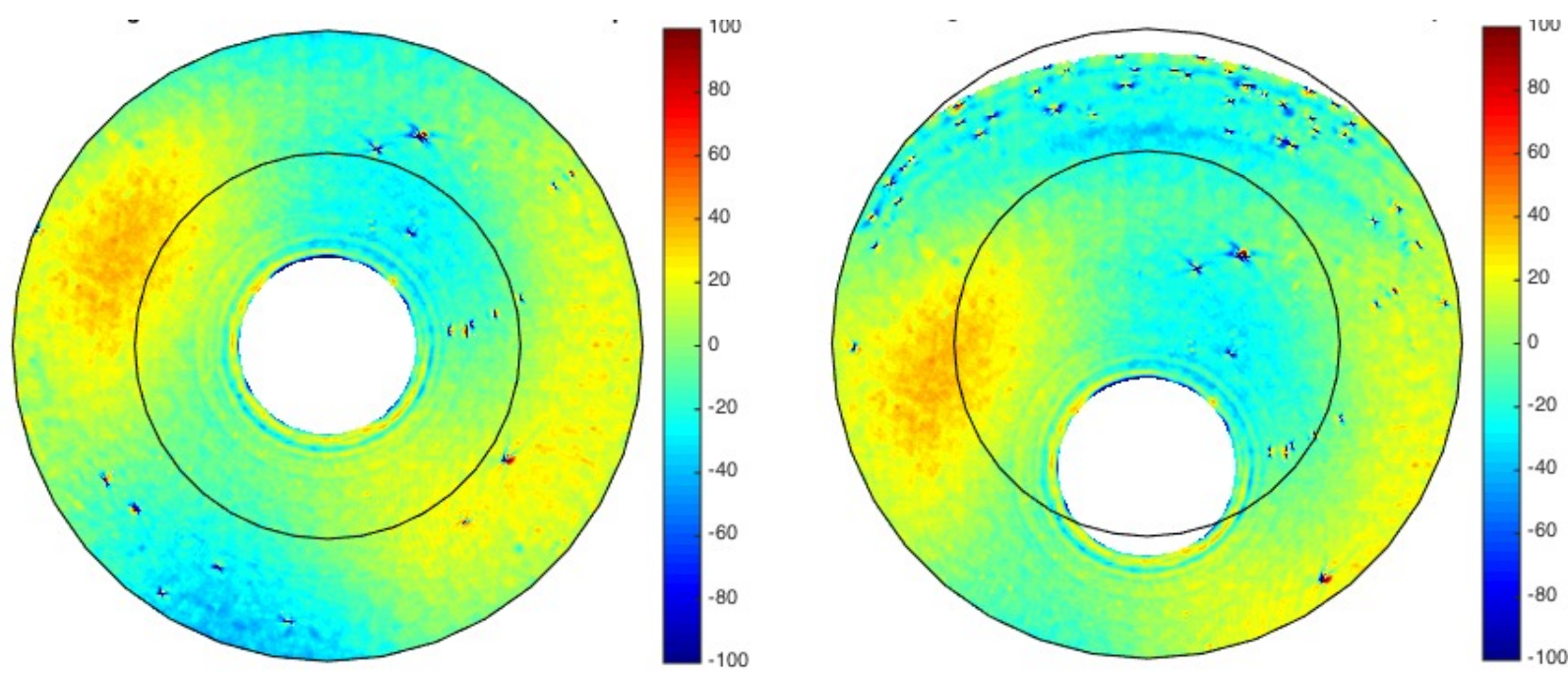

Figure 15. Synthetic map of M3 including 163 SPOTS maps, mapped onto the pupil for field angle $=0$ (left) and field angle $=1.75^{\circ}$ (right). The black circles indicate the inner and outer boundaries of the pupil. Vignetting by M2 is ignored.

All PSFs shown here include 0.6 arcsecond seeing, using the standard Kolmogorov model. We computed the PSF for seeing as the Fourier transform of the autocorrelation function $a$ of the complex field, a function of the separation vector $s$ in the pupil. The autocorrelation function is related to the phase structure function $\delta^{2}$ as $a(s)=\exp \left(-\delta^{2}(s) / 2\right)$, where $s$ is the separation vector in the pupil. We convolved this PSF with that of the mirror by multiplying their Fourier transforms.

PSFs are displayed with units of fraction of total energy per square arcsecond, so $\iint \operatorname{PSF} d \theta d \varphi=1$ when the angles $\theta$ and $\varphi$ are in arcseconds.

Where noted, the PSFs include the wide-angle scattering known as the aureole $\mathrm{e}^{[13],[14]}$ as well as the 0.6 arcsecond Kolmogorov seeing. We modeled the aureole as a constant $4 \times 10^{-5}$ of the energy per square arcsecond out to a radius $r=$ 5 arcsecond, and decreasing as $r^{-2}$ for $r>5$ arcsecond.

We first give results for PSFs that include 0.6 arcsecond Kolmogorov seeing plus the aureole. In order to isolate the impact of the crows' feet, we computed the PSF for three cases: perfect mirrors, M1 and M3 surface errors given by the interferometer maps only, and M1 and M3 given by the synthetic maps including SPOTS maps. M2 was ignored. Figure 16 shows the average radial profiles for the three cases, and Figure 17 shows the encircled energy. The surface errors present in the interferometer maps have a small effect on the PSF that extends to about $r=4$ arcsecond, limited by the sampling and resolution of the interferometers. Including the SPOTS maps transfers more energy from the core to the wings of the PSF. The fractional effect is small in the core but more significant in the wings. Compared with the PSF for perfect mirrors, the surface errors given by the interferometer maps decrease the central intensity by $6.3 \%$. The surface errors given by the synthetic maps decrease the central intensity by $7.8 \%$ relative to perfect mirrors. 

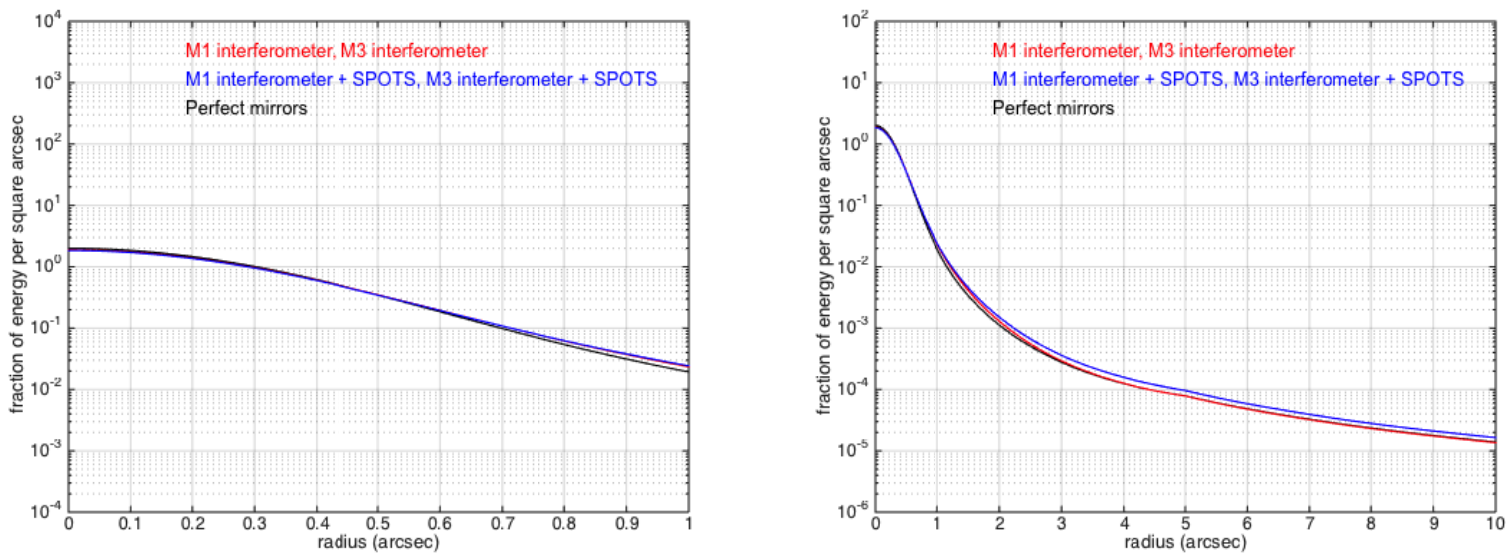

Figure 16. Average radial profiles of the PSFs for perfect mirrors, mirrors with surface errors given by the interferometer maps, and mirrors with surface errors given by the synthetic maps. All PSFs include 0.6 arcsecond seeing and the aureole. Both graphs show the same data but over different ranges.
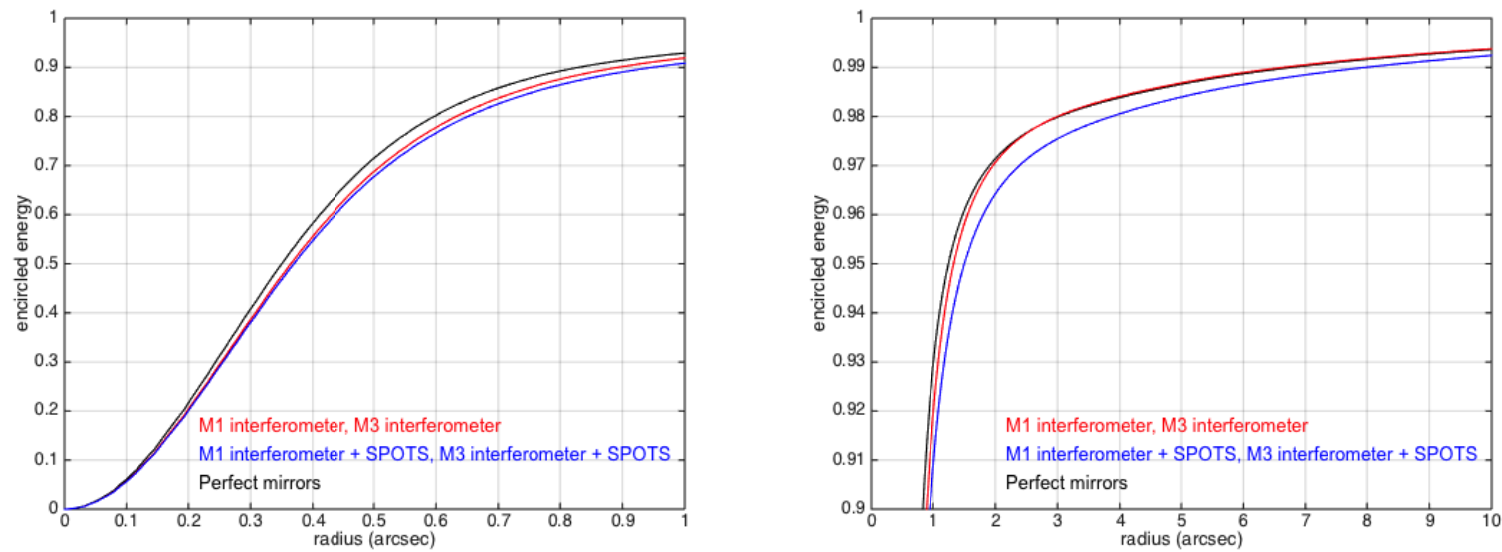

Figure 17. Encircled energy for the PSFs in Figure 16. Both graphs show the same data but over different ranges.

Table 2 lists the change in width and the change in brightness due to the addition of SPOTS maps, for the half-maximum point on the PSF and for several smaller fractions of the maximum extending into the wings of the PSF. The SPOTS maps have no effect on the full width at half max; with or without SPOTS maps the mirror surface error increases the FWHM from 0.6 arcsecond to 0.616 arcsecond. Adding the SPOTS maps increases the width by $1.3 \%$ at the $10^{-2}$ point and by $9 \%$ at the $10^{-4}$ point. Adding the SPOTS maps reduces the brightness in the core of the PSF by $1.4 \%$ at the center and by $1.5 \%$ at the half-max point, and increases the brightness in the wings by $4 \%$ at the $10^{-2}$ point $(r=1.05$ arcsecond $)$ and by $24 \%$ at the $10^{-4}$ point ( $r=3.46$ arcsecond). This brightening of the wings is the most significant effect of the crows' feet, and represents a modest increase in the faintest part of the PSF.

We also give results for PSFs that include only 0.6 arcsecond Kolmogorov seeing, with no aureole. Figure 18 shows the average radial profiles for the three cases, and Figure 19 shows the encircled energy. Again, the surface errors present in the interferometer maps have a small effect on the PSF. Adding the SPOTS maps transfers more energy from the core to the wings of the PSF.

With or without the aureole, including the SPOTS maps of crows' feet has a small effect on the energy lost from the core of the PSF. The change in central intensity due to crows' feet is the same with or without the aureole, but the effect on the wings is greater without the aureole. Removing the aureole accentuates the effect of crows' feet (or any other structure) on the wings of the PSF. 
Table 2. Width of the PSF at given fractions of the maximum, for the interferometer maps only and for the synthetic maps with SPOTS maps added; and change in brightness due to the addition of SPOTS maps, for the same fractions of the maximum. The PSFs include 0.6 arcsecond seeing and the aureole. The fractions of maximum refer to the PSF based on the interferometer maps.

\begin{tabular}{|c|c|c|c|}
\hline \multirow[b]{2}{*}{ amplitude } & \multicolumn{2}{|c|}{ full width to given amplitude } & \multirow{2}{*}{$\begin{array}{l}\text { change in } \\
\text { brightness due to } \\
\text { crows' feet }\end{array}$} \\
\hline & $\begin{array}{c}\text { M1 and M3 } \\
\text { interferometers only }\end{array}$ & $\begin{array}{l}\mathrm{M} 1 \text { and M3 } \\
\text { interferometers + } \\
\text { SPOTS }\end{array}$ & \\
\hline half max & $0.616 "$ & $0.616 ”$ & $-1.5 \%$ \\
\hline $0.1 \max$ & $1.212^{\prime \prime}$ & $1.216^{\prime \prime}$ & $-0.7 \%$ \\
\hline $0.01 \max$ & $2.106 "$ & $2.134 "$ & $4.1 \%$ \\
\hline $0.001 \max$ & $3.642 ”$ & $3.794 "$ & $14.9 \%$ \\
\hline $0.0001 \max$ & $6.910 "$ & $7.526 "$ & $24.5 \%$ \\
\hline
\end{tabular}
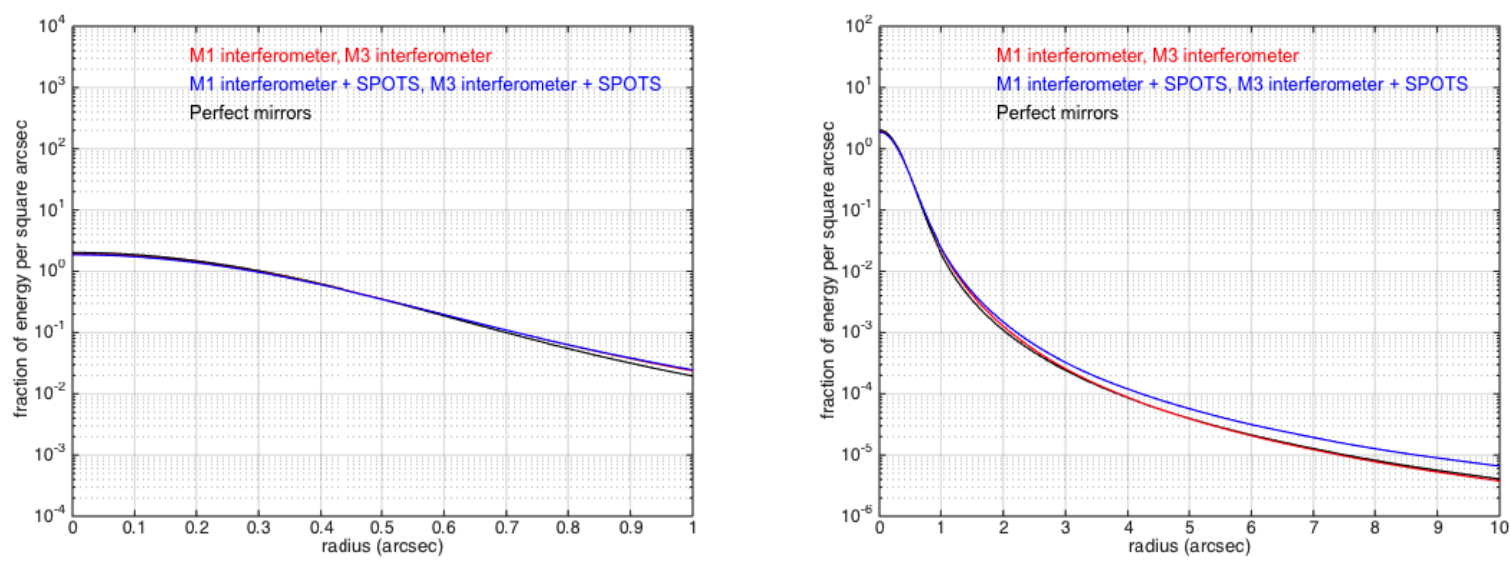

Figure 18. Average radial profiles of the PSFs for perfect mirrors, mirrors with surface errors given by the interferometer maps, and mirrors with surface errors given by the synthetic maps. All PSFs include 0.6 arcsecond seeing, with no aureole. Both graphs show the same data but over different ranges.
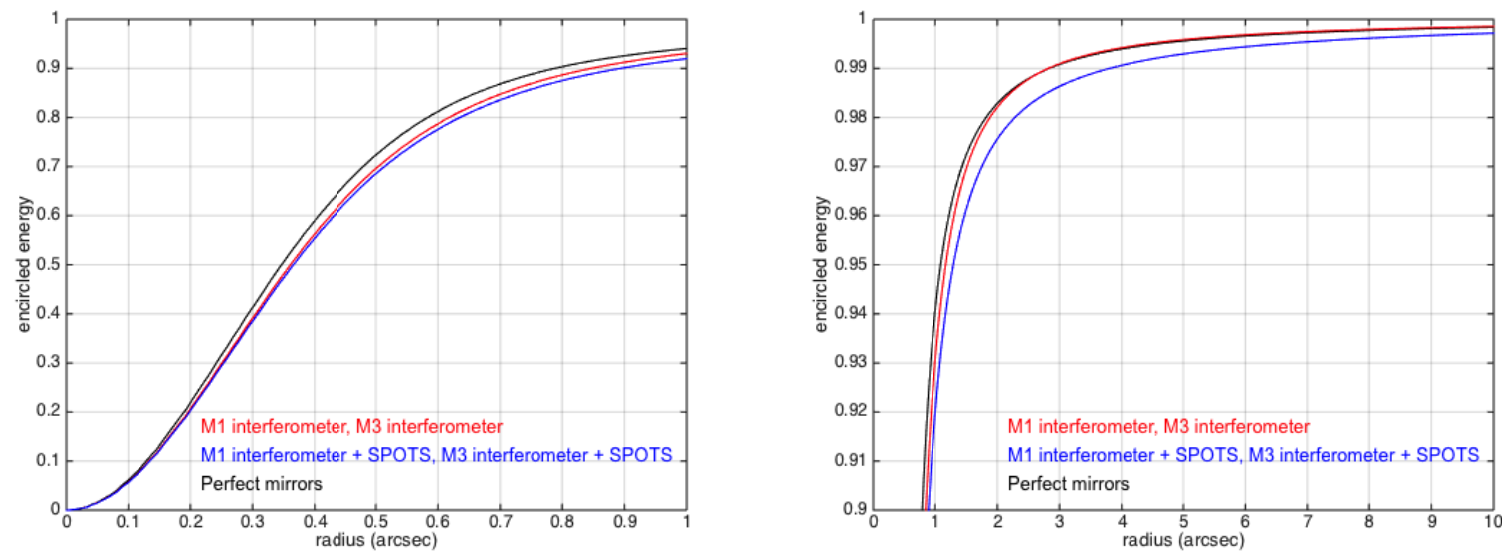

Figure 19. Encircled energy for the PSFs in Figure 18. Both graphs show the same data but over different ranges. 
Table 3 lists the change in width and the change in brightness due to the addition of SPOTS maps. Results are very similar to those including the aureole. The SPOTS maps have no effect on the full width at half max. Adding the SPOTS maps increases the width by $1.5 \%$ at the $10^{-2}$ point and by $8 \%$ at the $10^{-4}$ point. Adding the SPOTS maps reduces the brightness in the core of the PSF by $1.4 \%$ at the center and by $1.5 \%$ at the half-max point, and increases the brightness in the wings by $4 \%$ at the $10^{-2}$ point $\left(r=1.05\right.$ arcsecond) and by $29 \%$ at the $10^{-4}$ point $(r=3.24$ arcsecond $)$.

Table 3. Width of the PSF at given fractions of the maximum, for the interferometer maps only and for the synthetic maps with SPOTS maps added; and change in brightness due to the addition of SPOTS maps, for the same fractions of the maximum. The PSFs include 0.6 arcsecond seeing, with no aureole. The fractions of maximum refer to the PSF based on the interferometer maps.

\begin{tabular}{|c|c|c|c|}
\hline \multirow{2}{*}{ amplitude } & \multicolumn{2}{|c|}{ full width to given amplitude } & \multirow{2}{*}{$\begin{array}{c}\text { change in } \\
\text { brightness due to } \\
\text { crows' feet }\end{array}$} \\
\cline { 2 - 3 } & $\begin{array}{c}\text { M1 and M3 } \\
\text { interferometers only }\end{array}$ & $\begin{array}{c}\text { M1 and M3 } \\
\text { interferometers }+ \\
\text { SPOTS }\end{array}$ & $-1.5 \%$ \\
\hline half max & $0.616 ”$ & $0.616 ”$ & $-0.7 \%$ \\
\hline $0.1 \max$ & $1.212 ”$ & $1.216 ”$ & $4.0 \%$ \\
\hline $0.01 \max$ & $2.104 ”$ & $2.134 ”$ & $15.1 \%$ \\
\hline $0.001 \max$ & $3.624 ”$ & $3.772 ”$ & $28.9 \%$ \\
\hline $0.0001 \max$ & $6.488 ”$ & $7.014 ”$ & \\
\hline
\end{tabular}

These results show that including the high-resolution maps of crows' feet has little impact on the images. In parallel with the analysis described here, LSST staff performed independent PSF computations based on the same model of crows' feet that we presented. ${ }^{[15]}$ Their analysis included computing the normalized point source sensitivity (PSSN). The conclusion of this analysis is that the impact of crows' feet on the PSSN is well within the LSST system performance margins and will have negligible scientific impact.

\section{CONCLUSION}

The LSST primary-tertiary mirror has been completed and has been shown to give excellent performance and meet all specifications and requirements. Mirror Lab and LSST Project staff jointly validated the quality and performance of the mirror through measurements and analysis. This validation included a demonstration that both mirrors meet stringent figure accuracy specifications simultaneously. A simulated active-optics correction that takes account of the coordinated bending of both surfaces was used to guide the figuring of both mirrors and to demonstrate the simultaneous accuracy of both mirrors. Acceptance tests included interferometric measurements of the mirror surfaces, measurements of each mirror's radius of curvature and conic constant, measurements of the relative alignment of the two surfaces, and analysis of the impact on performance of small surface features that were not well resolved by the interferometers. Following these tests and analysis, the LSST Project accepted the mirror and it was delivered to the Project. Figure 20 shows the mirror prepared for shipment, and the mirror being transferred from the polishing cell to the transport frame.

\section{ACKNOWLEDGEMENTS}

This material is based upon work supported in part by the National Science Foundation through Cooperative Agreement Award No. AST-1227061 under Governing Cooperative Agreement 1258333 managed by the Association of Universities for Research in Astronomy (AURA), and the Department of Energy under Contract No. DEAC0276SF00515 with the SLAC National Accelerator Laboratory. Additional LSST funding comes from private donations, grants to universities, and in-kind support from LSSTC Institutional Members. 

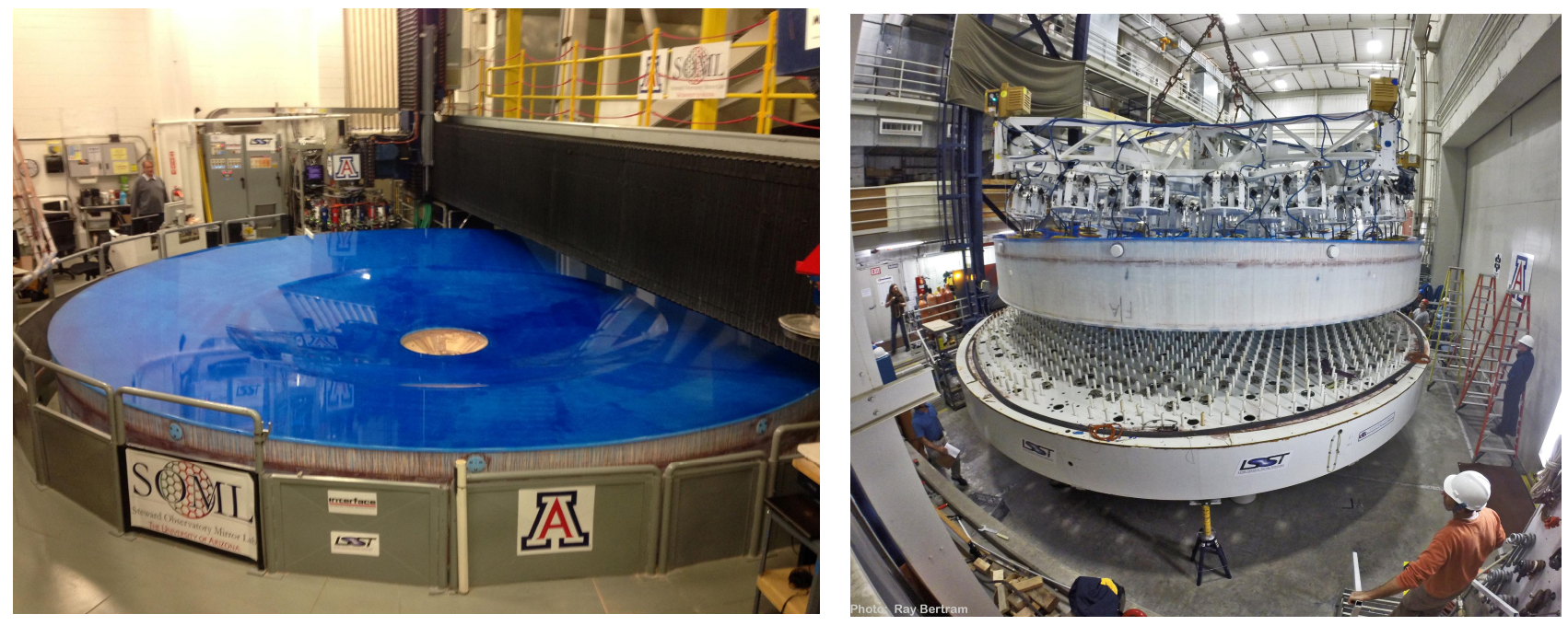

Figure 20. Left: LSST mirror covered with a protective film in preparation for delivery and shipment to its storage facility. Right: Mirror being lifted from its polishing cell prior to installation in the transport frame. The mirror is lifted with 36 vacuum pads acting through the porous film.

\section{REFERENCES}

[1] W. Gressler, J. DeVries, E. Hileman, D. R. Neill, J. Sebag, O. Wiecha, J. Andrew, P. Lotz and W. Schoening, "LSST telescope and site status", Proc. SPIE 9145, 91451A (2014).

[2] H. M. Martin, R. G. Allen, J. H. Burge, B. Cuerden, W. J. Gressler, W. Hubler, D. W. Kim, J. S. Kingsley, K. Law, P. A. Strittmatter, M. T. Tuell, S. C. West, C. Zhao and P. Zhou, "Manufacture of the combined primary and tertiary mirrors of the Large Synoptic Survey Telescope", Proc. SPIE 9151, 915125 (2014).

[3] H. M. Martin, S. P. Callahan, B. Cuerden, W. B. Davison, S. T. DeRigne, L. R. Dettmann, G. Parodi, T. J. Trebisky, S. C. West and J. T. Williams, "Active supports and force optimization for the MMT primary mirror", Proc. SPIE 3352, 412 (1998).

[4] H. M. Martin, R. G. Allen, B. Cuerden, S. T. DeRigne, L. R. Dettmann, D. A. Ketelsen, S. M. Miller, G. Parodi and S. Warner, "Primary mirror system for the first Magellan telescope", Proc. SPIE 4003, 2 (2000).

[5] H. M. Martin, R. G. Allen, J. H. Burge, L. R. Dettmann, D. A. Ketelsen, S. M. Miller and J. M. Sasian, "Fabrication of mirrors for the Magellan Telescopes and the Large Binocular Telescope", Proc. SPIE 4837, 609 (2003).

[6] P. Schechter, G. S. Burley, C. L. Hull, M. Johns, H. M. Martin, S. Schaller, S. A. Shectman and S. C. West, "Active optics on the Baade 6.5-m (Magellan I) Telescope", Proc. SPIE 4837, 619 (2003).

[7] H. M. Martin, B. Cuerden, L. R. Dettmann and J. M. Hill, "Active optics and force optimization for the first $8.4 \mathrm{~m}$ LBT mirror", Proc. SPIE 5489, 826 (2004).

[8] S. C. West, H. M. Martin, R. H. Nagel, R. S. Young, W. B. Davison, T. J. Trebisky, S. T. DeRigne and B. B. Hille, "Practical design and performance of the stressed lap polishing tool", Applied Optics, 33, 8094 (1994).

[9] D. W. Kim and J. H. Burge, "Rigid conformal polishing tool using non-linear visco-elastic effect", Opt. Express, 18, $2242(2010)$.

[10]D. W. Kim, H. M. Martin and J. H. Burge, "Calibration and optimization of computer-controlled optical surfacing for large optics", Proc. SPIE 8126, 812615 (2011).

[11] M. T. Tuell, J. H. Burge, B. Cuerden, W. Gressler, H. M. Martin, S. C. West and C. Zhao, "Final acceptance testing of the LSST monolithic primary/tertiary mirror", Proc. SPIE 9151, 91510W (2014).

[12] A. V. Maldonado, P. Su and J. H. Burge, "Development of a portable deflectometry system for high spatial resolution surface measurements", Applied Optics, 53, 4023 (2014).

[13] I. R. King, "The Profile of a Star Image", Pub. Astron. Soc. Pacific, 83, 199 (1971).

[14] R. Racine, "The Telescope Point-Spread Function”, Pub. Astron. Soc. Pacific, 108, 699 (1996).

[15] J. Sebag, W. Gressler, M. Liang, D. Neill, C. Araujo-Hauck, J. Andrew, G. Angeli, M. Cho, C. Claver, F. Daruich, E. Hileman, V. Krabbendam, G. Poczulp, R. Repp, G. Muller, O. Wiecha, B. Xin, K. Kenagy, H. M. Martin, M. T. Tuell and S. C. West, "LSST primary/tertiary monolithic mirror", Proc. SPIE 9906, paper 9906-126 (2016). 